\title{
Modeling and Analysis of the Influence of an Edge Filter on the Combining Efficiency and Beam Quality of a 10-kW-Class Spectral Beam-Combining System
}

\author{
Jun Ma ${ }^{1,2, *}$, Fan Chen ${ }^{1}$, Cong Wei ${ }^{1}$ and Rihong Zhu ${ }^{1,2}$ \\ 1 School of Electronic Engineering \& Optoelectronic Technology, Nanjing University of Science \& Technology, \\ Nanjing 210094, China; pananchenfan@163.com (F.C.); 115104000512@njust.edu.cn (C.W.); \\ zhurihong@njust.edu.cn (R.Z.) \\ 2 Key Laboratory of Advanced Solid-State Laser Technology, Ministry of Industry and Information \\ Technology, Nanjing University of Science \& Technology, Nanjing 210094, China \\ * Correspondence: majun@njust.edu.cn; Tel.: +86-8430-3376
}

Received: 6 May 2019; Accepted: 24 May 2019; Published: 27 May 2019

Featured Application: The constructed model is able to provide some theoretical instructions for the future design of a filter that is beneficial for achieving an optimal combining efficiency and beam quality for the 10-kW-class filter-based spectral beam combination system.

\begin{abstract}
Filter-based spectral beam combining (FSBC) is a promising power-scaling concept for high-power, broad-linewidth fiber lasers, as it relaxes the requirements for linewidth control and also the sizes of the individual beams. As the combining element in the FSBC system, the steep-edge filter plays a major role in achievement of the combining efficiency and the beam quality. In this case, we combine the uncorrelated surface roughness model and the combining efficiency model, and we conduct a comprehensive analysis of the effects of surface roughness, thickness error, and incident angle on the filter's optical properties and the combining efficiency, in order to determine the optimal configuration for the laser beam-combining system. The simulation results show a good agreement with the measured ones. Meanwhile, through the adoption of the angular spectrum theory, this paper has also conducted a preliminary analysis of the influence of the combining elements on the quality of the combined beam, and some theoretical instructions on the future design of the spectral beam-combining system are provided.
\end{abstract}

Keywords: laser beam combining; filters; scattering theory; multilayer design; combining efficiency

\section{Introduction}

In recent years, $\mathrm{Yb}$-doped fiber lasers (YDFLs) have been widely applied to the fields of scientific research and industrial production, owing to its advantages, which include a high conversion efficiency, a high beam quality, and excellent heat dissipation [1]. Benefiting from the development of large-mode-area (LMA) fibers and high-brightness semiconductor-based pump sources, the range of the output power achieved by the fiber lasers has been obviously improved [2-4]. Unfortunately, as they are limited by stimulated Brillouin scattering and undesirable thermo-optics, it is difficult to scale up the output power of a single fiber laser to higher power levels, and this would hardly meet the increasing demands of modern and technologically advanced industries [5-9].

Filter-based spectral beam combining (FSBC) is a promising approach to significantly increasing beam brightness, with the basic principle of multiplexing a certain number of single beams in the near and far fields through the use of a steep-edge filter [10-12]. As the key element in the FSBC system, the filter needs to suffer exposure to all of the laser radiation from each incident channel, 
and the optical performance of the edge filter has a significant influence on combining efficiency and beam quality. An edge filter with high reflectivity and a steep rising edge would not only achieve a high combining efficiency, but it would reduce the negative influences on the laser beam-combining system that are induced by the scattering light. Meanwhile, the steep rising edge of the filter is conducive to supporting the combination of more channels, so as to produce a higher combined output power. However, previous research has mainly focused on the effect of spectral broadening, and the relationship between the combining efficiency and the reflectance curve of the combining element has not been sufficiently discussed [11]. Hence, it is essential to conduct a comprehensive analysis on the filter's optical characteristics, especially for the filter used in a high-energy spectral beam-combining system.

In this contribution, we introduce a robust spectral beam by using a steep-edge filter to combine two high-power, broad-linewidth single beams. As for the combining element, the optical properties of the filter are closely related to the combining efficiency and the beam quality. By quoting and extending the uncorrelated surface roughness model as proposed by Eastman [13], a comprehensive discussion has been made, to analyze the effects of surface roughness, thickness error, and the angle of incidence on the filter's reflectivity and combining efficiency. The simulation results could predict the trends of the combination efficiency, and they can aid in the optimization of the FSBC system. Moreover, based on the angular spectrum theory, a preliminary study was undertaken, to establish the relationship between the surface roughness and the combined beam quality, which will be beneficial for the future fabrication of high-performance optical filters, using a 10-kW-class laser beam-combining system.

\section{Theory}

The formula for calculating the reflectivity of multilayer elements is commonly derived from the Fresnel formula and the iteration algorithm [14]. However, the experimental reflectivity is usually lower than the theoretical value, which can be attributed to three aspects: firstly, the film stack is usually considered to be homogeneous and smooth in simulation, while the roughness of each interface can result in significant losses in the reflectivity of the filters during the coating process [15]; secondly, when limited by the coating devices and manufacturing techniques, the thickness of the film is inaccurate, and the accumulated thickness errors have an obvious effect on the reflectivity. The last important factor is the deviation of the angle from the normal incidence, which causes a change in the optical path difference between the reflected lights. In this case, several optical models and modeling approaches to accurately calculating the reflectivity of multilayer optical coatings have been presented, such as uncorrelated surface roughness model, additive surface roughness model, and uncorrelated bulk inhomogeneity model [16-21]. After repeated supplementation and correction [22,23], the uncorrelated surface roughness model is now considered to be an effective and practical approach for academic research into the relationship between the surface roughness and reflectivity values of multilayer elements.

In this model, each interface is treated as being completely uncorrelated with the other interfaces in the multilayer coating, and some assumptions should be emphasized: (I) the root mean square (RMS) surface roughness, $\sigma$, is typically much less than a wavelength; (II) for the case of a small incidence angle, the polarization effect is not considered; (III) the function of the surface profile $f(x, y)$ is a random function, and its associated probability density is a Gaussian distribution; (IV) the absorption of the edge filter is not included in this model; (V) the substrate is considered to be semi-infinite, so that its reflectance can be ignored [21]; (VI) the roughness of each interface is treated as the same as the filter's.

The schematic diagrams of the edge filter used in the 10-kW-class FSBC system are shown in Figure 1a [11,24]. The filter consists of $m(m=268)$ dielectric layers, each having an index $n$ and a thickness $d$. With regard to considerations for increasing the threshold, $\mathrm{SiO}_{2}\left(n_{1}=1.433\right)$ and $\mathrm{ZrO}_{2}$ $\left(n_{2}=1.97\right)$ films are deposited in an alternating fashion on the fused silica substrate $\left(n_{s}=1.5\right)$. In this case, the filter has a high damage threshold $\left(>20 \mathrm{MW} / \mathrm{cm}^{2}\right)$ and a steep rising edge. The surface roughness $\sigma$ of each interface here is considered as the same as the filter's, which could be accurately 
measured by the Veeco white light profiler. Dozens of positions are measured and the average value is $0.85 \mathrm{~nm}$ [24]. Figure $1 \mathrm{~b}$ illustrates the profile of the rough surface, and for the case of a small incidence angle $\theta_{i}\left(\theta_{i}<6^{\circ}\right)$, the amplitudes of the incident wave $E_{0}{ }^{+}$, the reflected wave $E_{0}{ }^{-}$, and the transmitted wave $E_{\mathrm{s}}{ }^{+}$can be related through the following [21]:

$$
\left[\begin{array}{c}
E_{0}^{+} \\
E_{0}^{-}
\end{array}\right]=P\left[\begin{array}{c}
E_{s}^{+} \\
0
\end{array}\right]
$$

where $P$ is the characteristic matrix of the multilayered optical coatings and described as:

$$
P=S_{01} I_{01} T_{1} S_{12} I_{12} \cdots T_{m} S_{m s} I_{m s}=\left[\begin{array}{ll}
p_{1} & p_{3} \\
p_{2} & p_{4}
\end{array}\right]
$$

where $I_{i j}$ represents the propagation of the field from layer $i$ to layer $j$, and $T_{j}$ represents the propagation of the field across the $j_{t h}$ layer. The expressions of $I_{i j}$ and $T_{j}$ are given by:

$$
\begin{aligned}
& I_{i j}=\frac{1}{t_{i j}}\left[\begin{array}{cc}
1 & r_{i j} \\
r_{i j} & 1
\end{array}\right] \\
& T_{j}=\left[\begin{array}{cc}
e^{i \varphi_{j}} & 0 \\
0 & e^{-i \varphi_{j}}
\end{array}\right]
\end{aligned}
$$

where $r_{i j}$ and $t_{i j}$ are the Fresnel reflection coefficient and the Fresnel transmission coefficient, respectively. Under the condition of a small incidence angle $\theta_{i}$, they can be expressed as:

$$
\begin{aligned}
r_{s} & =\frac{n_{i} \cos \theta_{i}-n_{j} \cos \theta_{j}}{n_{i} \cos \theta_{i}+n_{j} \cos \theta_{j}} \\
r_{p} & =\frac{n_{i} \cos \theta_{j}-n_{j} \cos \theta_{i}}{n_{i} \cos \theta_{j}+n_{j} \cos \theta_{i}} \\
t_{s} & =\frac{2 n_{i} \cos \theta_{i}}{n_{i} \cos \theta_{i}+n_{j} \cos \theta_{j}} \\
t_{p} & =\frac{2 n_{i} \cos \theta_{i}}{n_{i} \cos \theta_{j}+n_{j} \cos \theta_{i}}
\end{aligned}
$$

where $\varphi_{j}$ is the optical phase thickness of layer $j$, and it is given as:

$$
\varphi_{j}=\frac{2 \pi n_{j} d_{j} \cos \theta_{j}}{\lambda}
$$

(a)

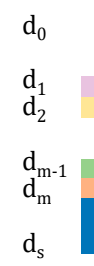

INCIDENT MEDIUM

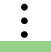

SUBSTRATE

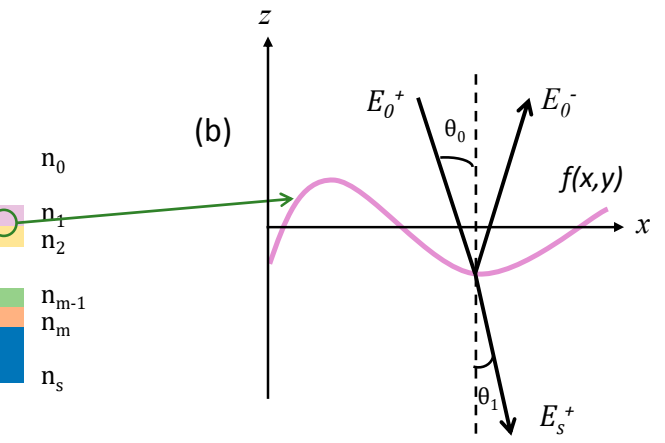

Figure 1. (a) Schematic diagrams of the steep-edge filters; (b) the profile of the rough surface [21,24]. 
The matrix $S$ represents the effect of the surface roughness, and it can be expressed as:

$$
\begin{aligned}
& S_{i j}=\frac{1}{\left(1-r_{i j}^{2}\right)}\left[\begin{array}{cc}
a-r_{i j}^{2} b & r_{i j}(b-a) \\
r_{i j}\left(b^{-1}-a^{-1}\right) & a^{-1}-r_{i j}^{2} b^{-1}
\end{array}\right] \\
& a=\exp \left\{i\left[\frac{2 \pi f(x, y)\left(n_{i} \cos \theta_{i}-n_{j} \cos \theta_{j}\right)}{\lambda}\right]\right\} \\
& b=\exp \left\{i\left[\frac{2 \pi f(x, y)\left(n_{i} \cos \theta_{i}+n_{j} \cos \theta_{j}\right)}{\lambda}\right]\right\}
\end{aligned}
$$

where $z=f(x, y)$ is the function of the surface profile, and its associated probability density $\omega(z)$ can be expressed as:

$$
w(z)=\frac{1}{\sqrt{2 \pi} \sigma} \exp \left(-\frac{z^{2}}{2 \sigma^{2}}\right)
$$

where $\sigma$ is the RMS surface roughness. Thus, the amplitude reflectance $r(\lambda)$ and the transmittance $t(\lambda)$ of the edge filter can be given as:

$$
\begin{gathered}
r(\lambda)=p_{2} / p_{1} \\
t(\lambda)=1 / p_{1}
\end{gathered}
$$

The radiant reflectance $R(\lambda)$ is the absolute square of the amplitude reflectance:

$$
R(\lambda)=r \cdot r^{*}
$$

If the angle of incidence is $\theta_{0}$ and the angle of refraction is $\theta_{s}$, the transmittance curve is deduced as follows:

$$
T(\lambda)=\left(n_{s} \cos \theta_{s} / n_{0} \cos \theta_{0}\right) \cdot t \cdot t^{*}
$$

For the case of the spectral beam-combining system, the filter as the combining element needs to overlap two beams at different wavelengths in both the near and far fields, and the optical reflectance curve $R(\lambda)$ of the filter will have a marked impact on the combining efficiency. Therefore, a combining efficiency model that is calculated from the laser spectrum is introduced into the uncorrelated surface roughness model, and the combining efficiency can be expressed as [11]:

$$
\eta=\frac{\sum_{\lambda_{1}}^{\lambda_{2}}\left[F_{2}(\lambda) * T(\lambda)\right]+\sum_{\lambda_{1}}^{\lambda_{2}}\left[F_{1}(\lambda) * R(\lambda)\right]}{\sum_{\lambda_{1}}^{\lambda_{2}} F_{1}(\lambda)+\sum_{\lambda_{1}}^{\lambda_{2}} F_{2}(\lambda)}
$$

where $F_{1}(\lambda)$ and $F_{2}(\lambda)$ represent the spectral curves of the incident laser beams, with $R(\lambda)$ being the reflectance curve of the edge filter and $\left(\lambda_{1}, \lambda_{2}\right)$ being the calculation interval.

\section{Simulation and Experimental Results}

The optical layout of the FSBC experiment is shown in Figure 2 [24]. Two beamlets emitting from the collimators with different wavelengths are combined by the edge filter. Each incident channel delivers an optical output power of about $5.5 \mathrm{~kW}$, and a combined output power of over $10 \mathrm{~kW}$ has been achieved. For the combining element, the filter needs to transmit a laser beam at $1090 \mathrm{~nm}$, and it needs to reflect the $1070 \mathrm{~nm}$ laser beam; thus, the combining efficiency and beam quality of the combined output beam are very relevant to the filter's reflectivity and the steepness of the rising edge. 


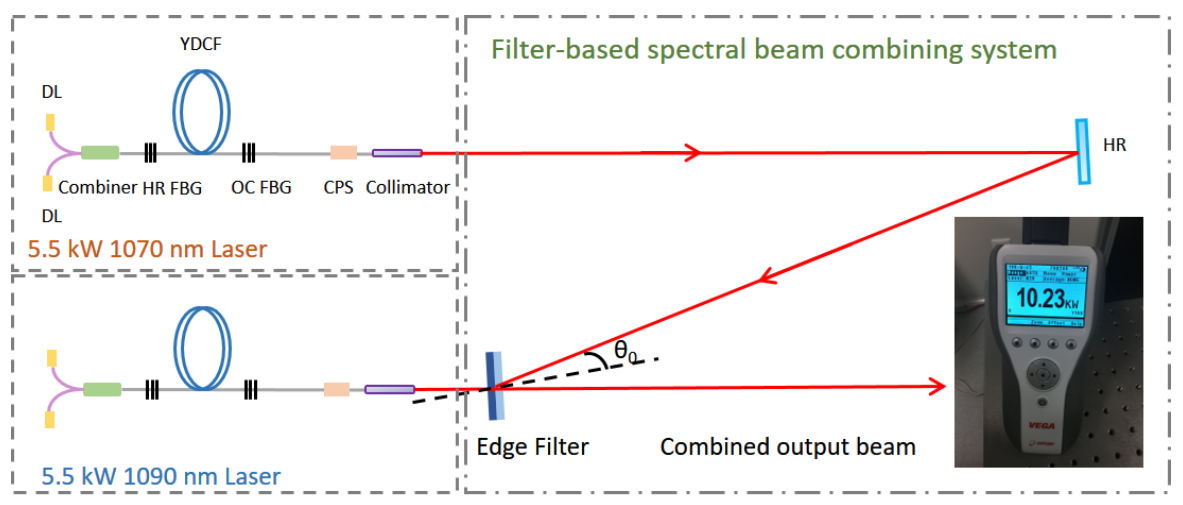

Figure 2. Experimental setup for a spectral beam formed from the combination of two high-power, broad-linewidth single beams. DL, diode laser; HR FBG, highly reflective fiber Bragg grating; OC FBG, output coupler fiber Bragg grating; YDCF, ytterbium-doped double-clad fiber; CPS, cladding power stripper; HR, high reflector [11,24].

\subsection{The Reflectance Curve of the Edge Filter}

To accurately measure the reflectance curve of the edge filter, we demonstrated a high-precision measurement system (Figure 3), including a broad-linewidth (1030-1110 nm)-amplified spontaneous emission (ASE) light source, a fiber collimator (FC), a polarization rotator, a convex lens with a 100 $\mathrm{mm}$ focal length, and an optical spectrum analyzer. Firstly, we measure the spectrum of the ASE light source in the absence of an edge filter and polarizer (as shown in the inset (a) of Figure 3). By inserting and rotating the polarization rotator, the collimated broad-linewidth laser is injected into a linear polarizer ( $p$ or $s)$. The laser with a pure polarization state is incident onto the surface of the edge filter, and then it is focused by a convex lens. The focus beam is collected by the probe of the spectrometer. Comparing the spectrum before and after the edge filter, the transmittance curve of the filter can be calculated accurately. With a low absorption coefficient, the absorption of the edge filter is negligible, and thus, the reflectance curve of the filter can be derived from the expression of the transmittance curve. On the other hand, by rotating the edge filter, the reflectance curves at various angles can also be measured (as shown in the inset (b) of Figure 3), which is of benefit for studying the relationship between the transmittance curves and the angle of incidence.
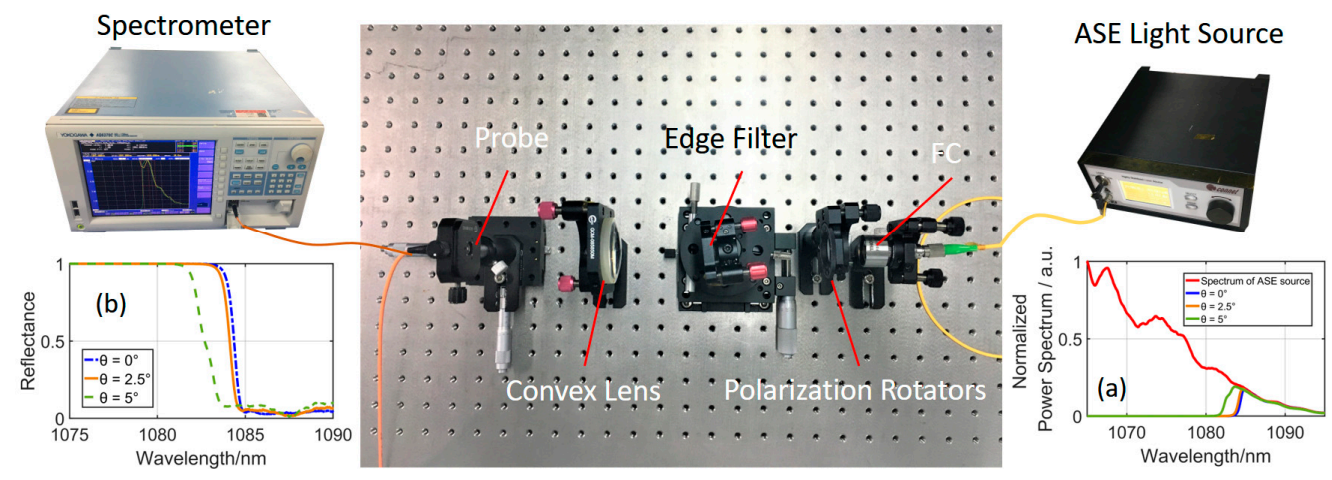

Figure 3. Test setup for measuring the reflectance curve of the edge filter. FC, fiber collimator; ASE light source, amplified spontaneous emission light source. Inset (a) shows the measured spectra; Inset (b) shows the experimental results of the reflectance curves under different incident angles $\left(0^{\circ}, 2.5^{\circ}\right.$, and $5^{\circ}$ ).

In general, there are several typical factors that influence the filter's optical characteristics, such as the surface roughness, the thickness error, and the angle of incidence. According to the uncorrelated surface roughness model (Equation (16)) and the structure of the edge filter, we calculated the reflectance curve of the edge filter under different surface roughness values. The simulation results for the normal 
incidence are shown in Figure 4a. With an increase in the surface roughness, a shift in the reflectance curve position is noticeable, and the rising edge tends to be gentler. The experimental result, as measured by the spectrometer, is indicated by the red line, and it shows a good agreement with the simulated results. Moreover, we have compared the model with the Thin Film Design Software TFCalc, and considered a multilayer coating with smooth surface and the thickness error not taken into account. The simulated reflectance curves for the normal incidence are shown in Figure $4 b, c$. The results indicate that the simulation derived from the proposed model agrees well with those obtained with the TFCalc, which proves the reliability of the model.
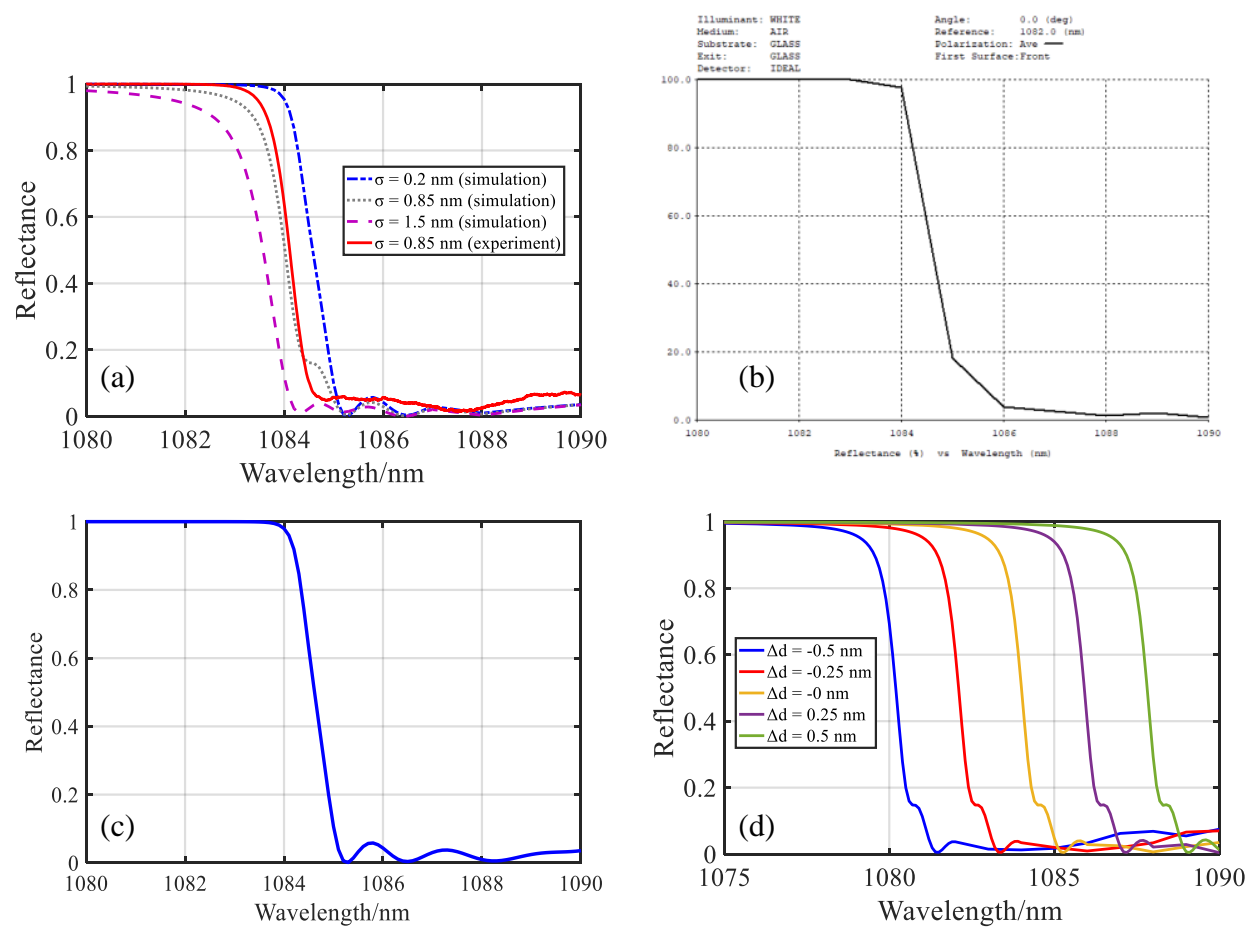

Figure 4. (a) The reflectance curves of the edge filter under different surface roughness values. The simulation results of the reflectance curve of the edge filter with smooth surface, obtained with: (b) TFCalc; (c) uncorrelated surface roughness model. (d) The reflectance curve of the edge filter versus the thickness error.

Except for the surface roughness, we also calculated the thickness error of the multilayer. A system of 268 dielectric layers was considered, each having the same variations from $-0.5 \mathrm{~nm}$ to 0.5 $\mathrm{nm}$, and the surface roughness was set to $0.85 \mathrm{~nm}$. To treat the case of normal incidence, the reflectance curves of the edge filter with respect to the thickness error are shown in Figure 4d, in which only a shift in the position of the reflectance curve occurs, and where the thickness error does not affect the steepness of the rising edge.

It should be noted that the thickness errors on each layer always occur randomly during the coating process. In this case, we also conducted simulations on the reflectance curve of the edge filter, with various random errors on each layer of the stack. Figure 5 shows the simulation results, and it is evident that the random errors of the thickness not only affect the position, but the steepness of the curve. Meanwhile, the magnitude of the random error aids in the enhancement of the fluctuation range of the curve, which in turn decreases the stability and reliability of the laser beam-combining system. Hence, random errors in the thickness for such a filter should be limited to within $0.3 \%$, so as to obtain a high combination efficiency and a stable output power level. 

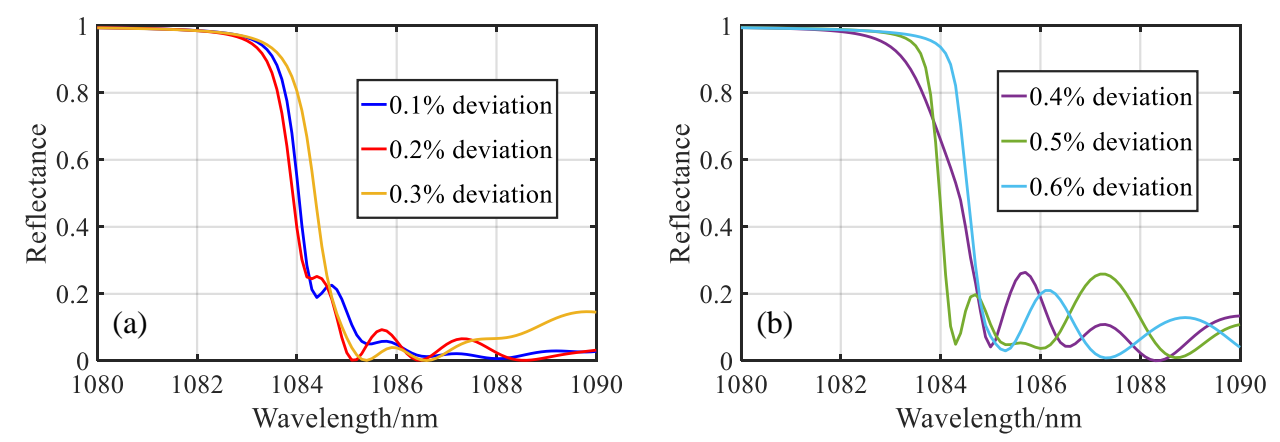

Figure 5. The reflectance curves of the edge filter with various random errors on each layer of the stack: (a) $0.1 \% \sim 0.3 \%$; (b) $0.4 \% \sim 0.6 \%$.

Based on the Fresnel equation and Snell's law, we extended the uncorrelated surface roughness model to calculate the filter's reflectivity under the circumstances of a small incident angle. Consider a multilayer coating with rough surfaces, as above $(\sigma=0.85 \mathrm{~nm})$, with the thickness error not taken into account. The simulation results (the solid lines) and the experimental results (the dotted line) of the relationship between the reflectance and incident angle for the two cases of polarization are shown in Figures $6 a$ and 6 , respectively. As can be clearly seen in the Figure 6 , the reflectance curve of the edge filter only moves toward short wavelengths, and the steepness of the rising edge is irrelevant to the incident angle. The simulations are consistent with the experiments results, verifying the accuracy and validity of the model.
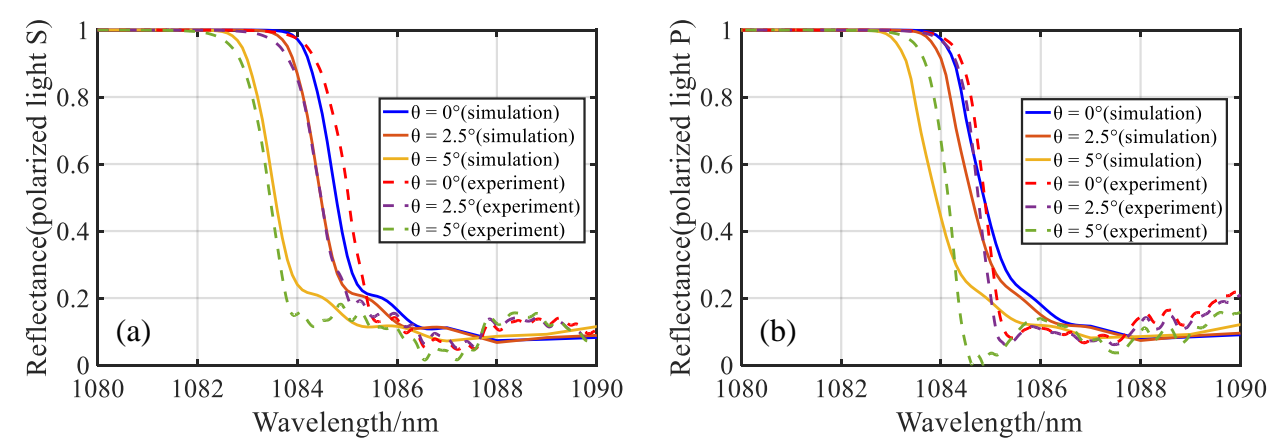

Figure 6. The simulation and experimental results of the reflectance curves under different incident angles: (a) $s$ polarization; (b) $p$ polarization.

\subsection{Effect of the Filter Optical Characteristics on the Combining Efficiency}

The deduced equations (Equations (16)-(18)) show that the spectra of the incident channels and the reflectance curve of the edge filter play a dominant role in achieving the combining efficiency. According to the above discussion and analysis, the reflectance curve is affected by the surface roughness, the thickness error, and the angle of incidence, and thus, a further discussion and analysis was included, to introduce the impacts of these factors on the combining efficiency. Figure 7a shows the measured normalized spectrum from a $1070 \mathrm{~nm}$ laser and a $1090 \mathrm{~nm}$ laser at full power. The broad linewidth of each channel (The full width at half maximum is over $4 \mathrm{~nm}$ ) influences the steepness of the reflectance curve, and the rising edge of the filter must be within the range of $1080 \mathrm{~nm}-1085 \mathrm{~nm}$, in order to achieve a high efficiency of combination. The simulation results of the combining efficiency as a function of the surface roughness are shown in Figure $7 \mathrm{~b}$. As expected, a greater surface roughness results in an increase of the scattering, and this subsequently leads to a decline in the combining efficiency. Apart from the aspect of scattering, the enhancement of the surface roughness also causes a shift in the position of the reflectance curve, and the rising edge tends to be gentler, which further aggravates the decline in the combining efficiency. Therefore, the combining efficiency shows a significant negative correlation with the surface roughness, especially when the surface roughness is more than $1 \mathrm{~nm}$. In 
general, the combining efficiency of a valid beam combination system should exceed $94 \%$, and in this case, the surface roughness of an edge filter, when used in a 10-kW-class FSBC system, should be within $1.2 \mathrm{~nm}$.
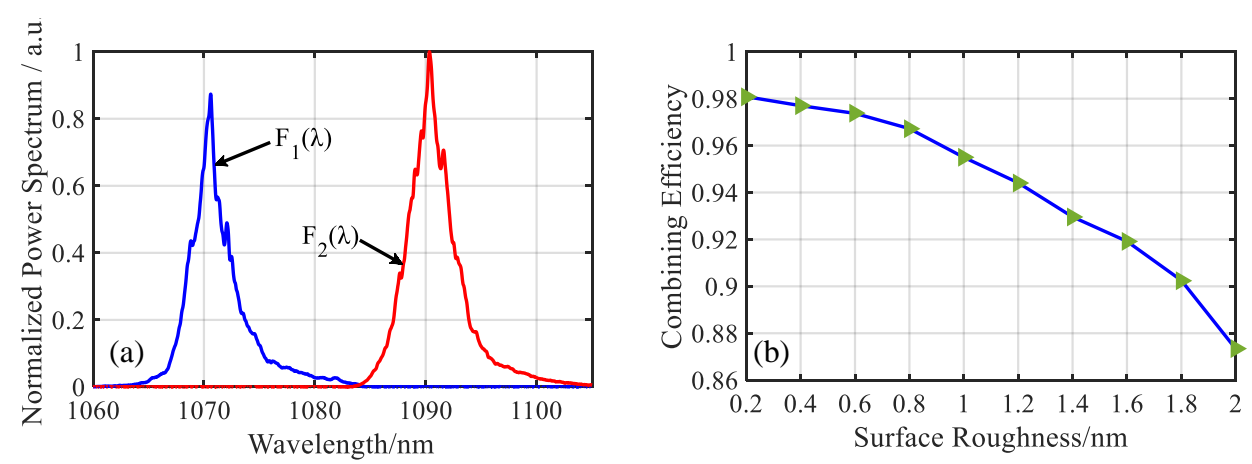

Figure 7. (a) The measured normalized spectra of the $1070 \mathrm{~nm}$ laser (blue line) and the $1090 \mathrm{~nm}$ laser (red line). (b) The combining efficiency as a function of the surface roughness.

Figure 8a shows the relationship between the combining efficiency and the thickness error. When the thickness error lies in the range of $0-0.25 \mathrm{~nm}$, the combining efficiency is almost constant, and the maximum value is $97 \%$. In other cases, the combining efficiency shows a significant negative correlation with the thickness error, especially when the error is greater than $0.25 \mathrm{~nm}$. For the case of the incident angle, an obvious decrease in the combining efficiency could be observed with the increasing incident angle (shown in Figure 8b). Hence, to achieve a high combining efficiency, the thickness error of such an edge filter should be limited to within $0.25 \mathrm{~nm}$, and a small incident angle is necessary. We have conducted a spectral beam-combining experiment with an incident angle of $2.5^{\circ}$, and the measured combining efficiency at full power is $95.8 \%$, which is consistent with the simulation result.
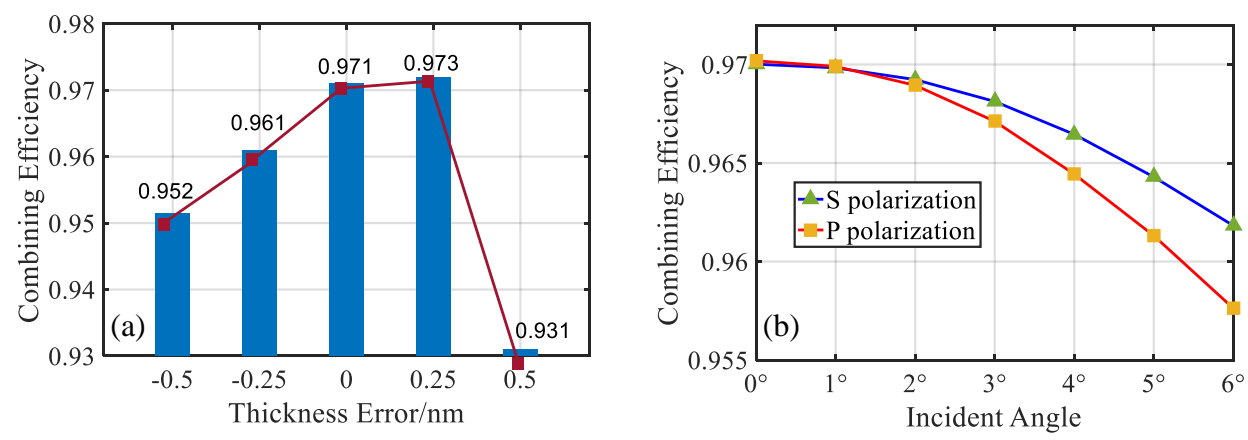

Figure 8. (a) The combining efficiency as a function of the thickness error. (b) The combining efficiency as a function of the incident angle. The blue line represents the s-polarized light, and the red line represents the $p$-polarized light.

\subsection{Effect of the Filter Surface Roughness on the Combined Beam Quality}

Beam quality is another important evaluation index for the design of the FSBC system, and the edge filter as the combining element also has a significant effect on this parameter. In our study, we also developed a preliminary analysis on the relationship between the filter's surface roughness and the combined beam quality.

\subsubsection{The Ideal Gaussian Beam}

We first assume that two laser individual channels operating at $1070 \mathrm{~nm}$ and $1090 \mathrm{~nm}$, respectively, are combined via an edge filter, and that their far-field intensity profiles have an ideal Gaussian 
distribution. According to Equations (14) and (15), the complex amplitudes of the reflected laser and transmitted laser can be expressed as:

$$
\begin{aligned}
& E_{r}\left(x_{r}, y_{r}, 0\right)=r * E_{0}\left(x_{0}, y_{0}, 0\right) \\
& E_{t}\left(x_{t}, y_{t}, 0\right)=t * E_{1}\left(x_{1}, y_{1}, 0\right)
\end{aligned}
$$

where $E_{0}$ and $E_{1}$ are the amplitudes of the incident lasers. Based on the angular spectrum theory, the complex amplitudes of the beams at a propagation distance $z$ are obtained as:

$$
\begin{aligned}
E z_{0}(x, y, z)= & \iiint \int E_{0}\left(x_{0}, y_{0}, 0\right) * \exp \left(j \frac{2 \pi z}{\lambda} \sqrt{1-\lambda^{2} f_{x}^{2}-\lambda^{2} f_{y}^{2}}\right) \\
& * \exp \left\{j 2 \pi\left[f_{x}\left(x-x_{0}\right)+f_{y}\left(y-y_{0}\right)\right]\right\} d f_{x} d f_{y} d x_{0} d y_{0} \\
E z_{1}(x, y, z)=\quad & \iiint \int E_{1}\left(x_{1}, y_{1}, 0\right) \exp \left(j \frac{2 \pi z}{\lambda} \sqrt{1-\lambda^{2} f_{x}^{2}-\lambda^{2} f_{y}^{2}}\right) \\
& * \exp \left\{j 2 \pi\left[f_{x}\left(x-x_{1}\right)+f_{y}\left(y-y_{1}\right)\right]\right\} d f_{x} d f_{y} d x_{1} d y_{1} \\
E z_{r}(x, y, z)=\quad & \iiint \int E_{r}\left(x_{r}, y_{r}, 0\right) \exp \left(j \frac{2 \pi z}{\lambda} \sqrt{1-\lambda^{2} f_{x}^{2}-\lambda^{2} f_{y}^{2}}\right) \\
& * \exp \left\{j 2 \pi\left[f_{x}\left(x-x_{r}\right)+f_{y}\left(y-y_{r}\right)\right]\right\} d f_{x} d f_{y} d x_{r} d y_{r} \\
E z_{t}(x, y, z)= & \iiint \int E_{t}\left(x_{t}, y_{t}, 0\right) \exp \left(j \frac{2 \pi z}{\lambda} \sqrt{1-\lambda^{2} f_{x}^{2}-\lambda^{2} f_{y}^{2}}\right) \\
& * \exp \left\{j 2 \pi\left[f_{x}\left(x-x_{t}\right)+f_{y}\left(y-y_{t}\right)\right]\right\} d f_{x} d f_{y} d x_{t} d y_{t}
\end{aligned}
$$

where $f_{x}$ and $f_{y}$ are the spatial frequencies in the $x$ and $y$ directions, respectively, and the small incident angle $\left(2.5^{\circ}\right)$ allows us to ignore the effects of this angle on the complex amplitude. The purpose of this laser beam combination is to overcome the limits that are set by nonlinear optical processes and to scale up the output power. In this case, the encircled power in the bucket (PIB), which is defined as the ratio of the actual energy within a circle, as determined by the "diffraction-limited bucket" as being the ideal energy level within the same bucket, is treated as a classical parameter for the evaluation of the laser beam quality. The PIB expression is:

$$
\begin{aligned}
E z_{t}(x, y, z)= & \quad \iiint \int E_{t}\left(x_{t}, y_{t}, 0\right) \exp \left(j \frac{2 \pi z}{\lambda} \sqrt{1-\lambda^{2} f_{x}^{2}-\lambda^{2} f_{y}^{2}}\right) \\
& * \exp \left\{j 2 \pi\left[f_{x}\left(x-x_{t}\right)+f_{y}\left(y-y_{t}\right)\right]\right\} d f_{x} d f_{y} d x_{t} d y_{t}
\end{aligned}
$$

The numerical simulations of the PIB versus the surface roughness at a distance of $500 \mathrm{~mm}$, based on Equations (21)-(25), are shown in Figure 9a. As expected, the surface roughness of the filter has a negative influence on the combined beam quality, and the PIB gradually decreases as the surface roughness increases, which is especially obvious in the case of the transmitted laser. When the surface of the filter is smooth, both the reflected laser and the transmitted laser show a typical Gaussian distribution (Figure 9b). However, with the increase in surface roughness, the ratio of scattered beam gradually increases in the combined laser beams, which reduces the power focusability for both cases. Worse still, due to the influence of scattering, the far-field intensity profiles of the reflected and transmitted lasers (Figure 9c,d) typically have a non-Gaussian distribution, and this could result in the degradation of the combined beam quality (Figure 9e).

Meanwhile, based on the simulation results shown in Figure 10a, the propagation distance $Z$ will enhance the effects of scattering, and this further reduces PIB. Figure 10b-f shows the far-field intensity profiles of the combined beam under different propagation distances. With an increase in the propagation distance, the intensity distribution of the combined laser beams gradually drift off from the Gaussian distribution, and a significant deterioration in the combined beam quality is observed. Hence, for a combining element with a large surface roughness, the laser beam combination system is present only within a limited available irradiation range. 

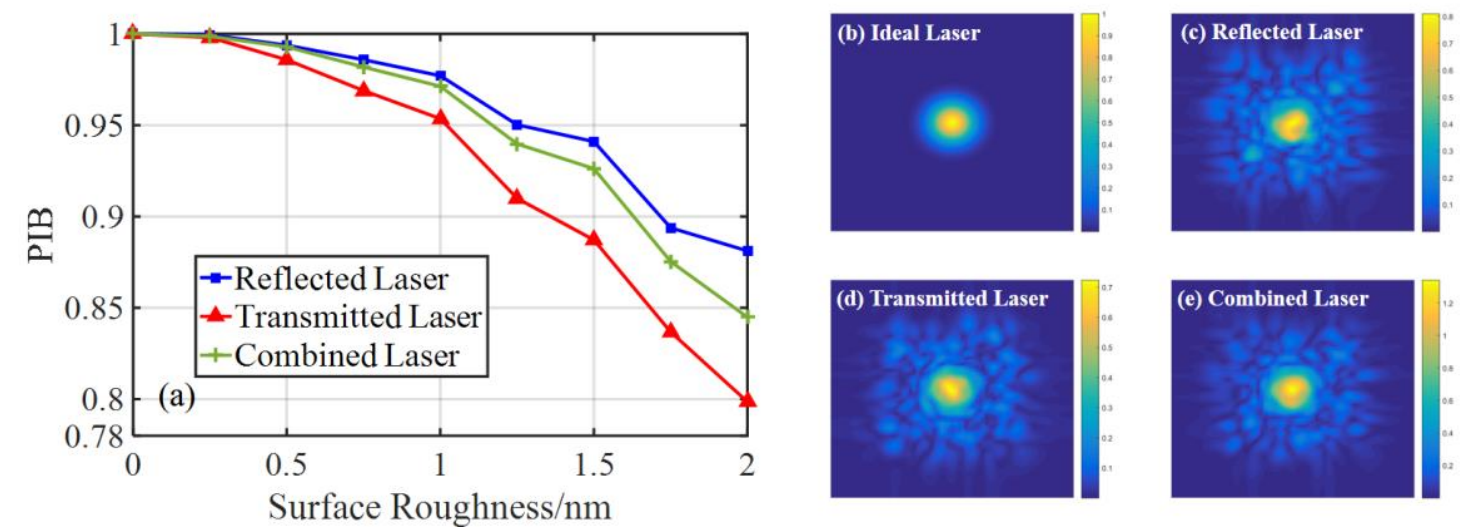

Figure 9. (a) The encircled power versus surface roughness at a distance of $500 \mathrm{~mm}$. The intensity profile of: (b) the ideal laser (surface roughness $\sigma=0 \mathrm{~nm}$ ); (c) the reflected laser (surface roughness $\sigma=2 \mathrm{~nm}$ ); (d) the transmitted laser (surface roughness $\sigma=2 \mathrm{~nm}$ ), and (e) the combined laser (surface roughness $\sigma=2 \mathrm{~nm}$ ), all at a distance of $500 \mathrm{~mm}$.

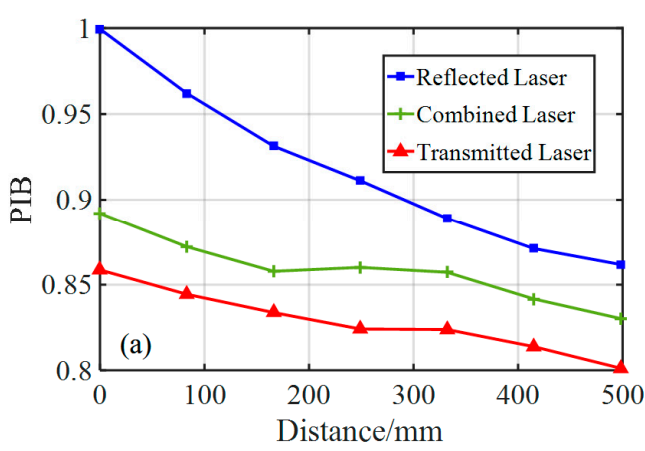

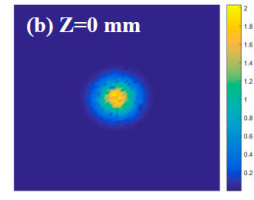

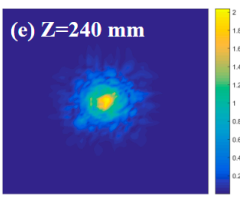

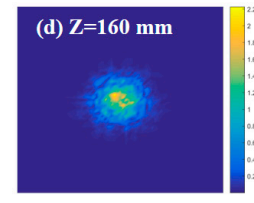
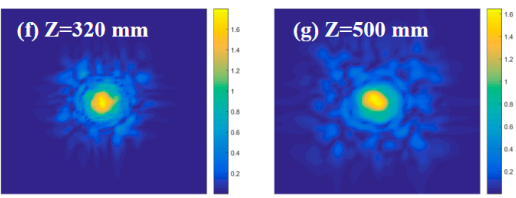

Figure 10. (a) The encircled power versus the propagation distance, with a surface roughness of $2 \mathrm{~nm}$. The intensity profiles of the combined beam at different propagation distances $Z$; (b) $Z=0 \mathrm{~mm}$; (c) $Z=80 \mathrm{~mm}$; (d) $Z=160 \mathrm{~mm}$; (e) $Z=240 \mathrm{~mm}$; (f) $Z=320 \mathrm{~mm}$; (g) $Z=500 \mathrm{~mm}$.

\subsubsection{The Multimode Gaussian Beam}

Generally, the multimode lasers that emerge from the resonator can be completely described as superpositions of the Hermite-Gaussian or Laguerre-Gaussian modes [25-27]. However, the accuracy of the modal decomposition technology is limited, and the expression of the complex amplitude is rather complex, especially for the beamlets that are achieved by the power combination of several laser oscillators. In this case, a large amount of work is theoretically needed when using the FSBC system, to obtain a precise expression for the multimode lasers. Fortunately, the PHASICS SID4 wave-front sensors, which are able to monitor the complex amplitudes of the beamlets in real time, provide a simple and reliable method for obtaining the complex amplitudes of the multimode lasers. These measurement results are shown in Figure 11.

$I_{0}$ and $I_{1}$ are the normalized intensities of the incident lasers, and are expressed as:

$$
\begin{aligned}
& I_{1}=\frac{I_{1}}{\max \left(I_{1}\right)} \\
& I_{0}=\frac{I_{0}}{\max \left(I_{1}\right)}
\end{aligned}
$$

where $\varphi_{0}$ and $\varphi_{1}$ are the phases of the incident lasers. Hence, the complex amplitudes of the incident lasers can be expressed as:

$$
E_{0}=\sqrt{I_{0}} \exp \left(-i \varphi_{0}\right)
$$




$$
E_{1}=\sqrt{I_{1}} \exp \left(-i \varphi_{1}\right)
$$

When Equations (28) and (29) are substituted into Equations (19) and (20), the relationship between the beam quality and the surface roughness at a distance of $500 \mathrm{~mm}$ can be potentially developed. The resulted encircled power distributions are shown in Figure 12, which shows a trend similar to that of an ideal Gaussian beam. The insets show the intensity profiles of the combined laser under different surface roughness values. As expected, the ratio of the scattered beam is gradually enhanced as the surface roughness increases, which leads to a deterioration in beam quality.
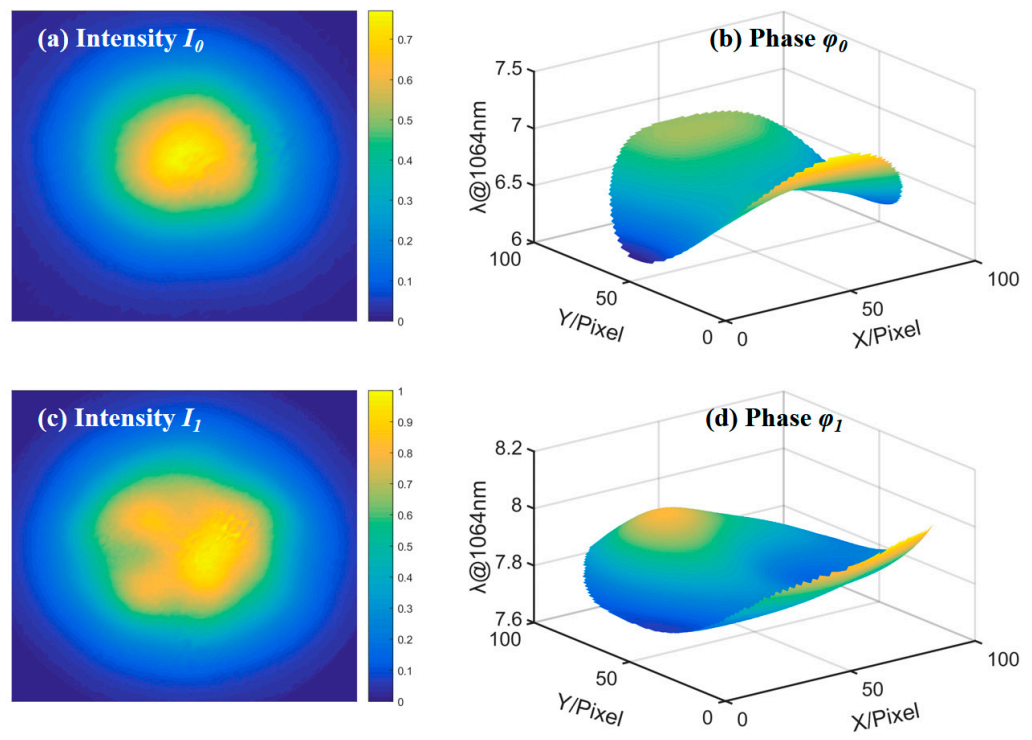

Figure 11. The measured complex amplitudes of the incident lasers.

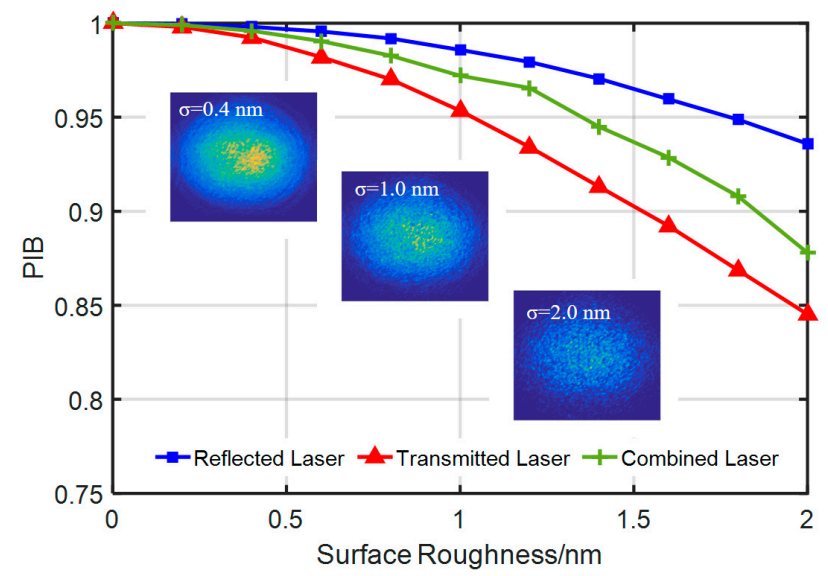

Figure 12. The encircled power versus surface roughness at a distance of $500 \mathrm{~mm}$. The insets are the intensity profiles of the combined laser under different surface roughness values.

On the other hand, the effect of the propagation distance on the PIB was also calculated. As shown in Figure 13, the encircled power displays a decreasing trend, with an increase in the propagation distance, and it is similar to that in the case of the single-mode lasers. The insets display the intensity profiles of the combined beam under different propagation distances, from which the enhancement of the scattering could be clearly observed. In this case, it is necessary to maintain the smoothness of the edge filter, which is not only beneficial for achieving combined beams with high power and good quality, but also helpful for enhancing the effective working distance of the combining system. 


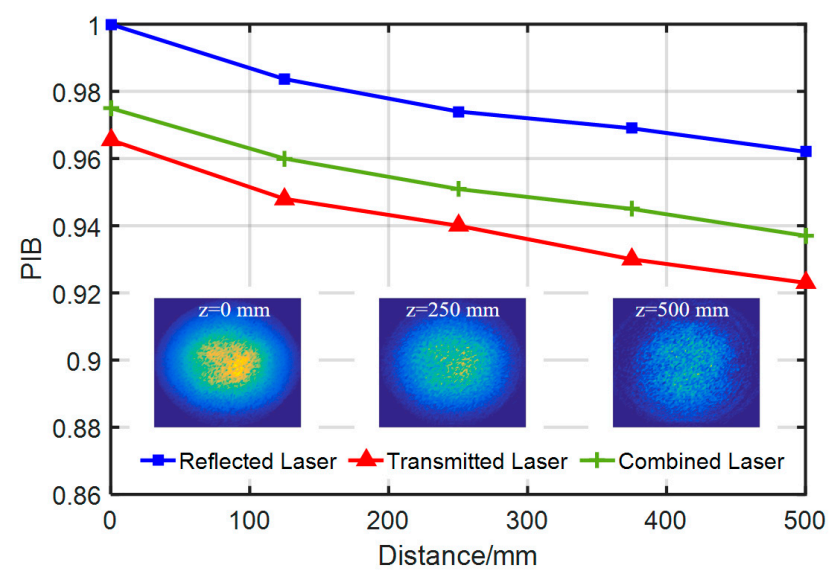

Figure 13. The encircled power versus propagation distance, with a surface roughness of $0.85 \mathrm{~nm}$. The insets display the intensity profiles of the combined beam at different propagation distances.

\section{Conclusions}

In this paper, we constructed an analytical model to discuss the impacts of a filter on the combining efficiency and the beam quality within an FSBC scheme. Via adopting the uncorrelated surface roughness model, we made a detailed analysis of the relationship between the combining efficiency and the optical characteristics of the edge filter. To achieve an optimal combining efficiency, the surface roughness of the edge filter that is used in the FSBC system should be less than $1.2 \mathrm{~nm}$, and the thickness error should be limited to within $0.25 \mathrm{~nm}$. Meanwhile, the combining efficiency shows a significant negative correlation with the incident angle, for both $s$ and $p$ polarization cases.

Furthermore, based on the angular spectrum theory, the effect of different surface roughness values for the filter (in terms of the combined beam quality) was briefly discussed. The simulation results show that the scattering that is induced by the surface roughness reduces the focusing power of the incident lasers, resulting in a degradation of the combined beam quality. Worse still, the propagation distance will enhance the effects of scattering, and further reduce the PIB. In this case, selecting an edge filter with a minimum surface roughness not only results in a better combined beam quality, but also enhances the effective working distance of the combining system. Even though the example considered here is an near-infrared edge filter, the analysis model is still suitable and useful for various multilayer elements with different surface roughness values.

Author Contributions: This work was realized by the collaboration of all authors. R.Z. guided the research direction and proposed the revising suggestions of manuscript. J.M. and F.C. designed the algorithms, carried the simulation, performed the experiments, and analyzed the experimental data. C.W. offered help for the simulations. J.M. wrote the paper. All authors read and approved the final manuscript.

Funding: This work was supported by the National Natural Science Foundation of China (NSFC) (No. 61575095, 61875087, and 11761131015), Sino-German Cooperation Research Group (No. GZ1391)

Acknowledgments: The authors are very grateful to Qing Wang for his help with the experiments.

Conflicts of Interest: The authors declare no conflict of interest.

\section{References}

1. Wirth, C.; Schmidt, O. $2 \mathrm{~kW}$ incoherent beam combining of four narrow-linewidth photonic crystal fiber amplifiers. Opt. Express 2009, 17, 1178-1183. [CrossRef]

2. Zervas, M.N.; Codemard, C.A. High power fiber lasers: A Review. IEEE J. Sel. Top. Quantum Electron. 2014, 20, 0904123. [CrossRef]

3. Limpert, J.; Röser, F. The rising power of fiber lasers and amplifiers. IEEE J. Sel. Top. Quantum Electron. 2007, 13, 537-545. [CrossRef]

4. Jauregui, C.; Otto, H.J. Optimizing high-power Yb-doped fiber amplifier systems in the presence of transverse mode instabilities. Opt. Express 2016, 24, 7879-7892. [CrossRef] [PubMed] 
5. Cherif, R.; Zghal, M. Characterization of stimulated Brillouin scattering in small core microstructured chalcogenide fiber. Opt. Commun. 2012, 285, 341-346. [CrossRef]

6. Loranger, S.; Lambin-Iezzi, V. Stimulated Brillouin scattering in ultra-long distributed feedback Bragg gratings in standard optical fiber. Opt. Lett. 2016, 41, 1797-1800. [CrossRef] [PubMed]

7. Li, Q.; Zhang, H. Stimulated Raman scattering threshold for partially coherent light in silica fibers. Opt. Express 2015, 23, 28438-28448. [CrossRef] [PubMed]

8. Sophie, A.; Patrick, M. Thermal effects on the photoelastic coefficient of polymer optical fibers. Opt. Lett. 2016, 41, 2517-2520. [CrossRef] [PubMed]

9. Mallek, D.; Kellou, A. Instabilities in high power fiber lasers induced by stimulated Brillouin scattering. Opt. Commun. 2013, 308, 130-135. [CrossRef]

10. Schmidt, O.; Wirth, C. Spectral beam combination of fiber amplified ns-pulses by means of interference filters. Opt. Express 2009, 17, 22974-22982. [CrossRef]

11. Chen, F.; Ma, J. Coupling efficiency model for spectral beam combining of high-power fiber lasers calculated from spectrum. Appl. Opt. 2017, 56, 2574-2579. [CrossRef]

12. Regelskis, K.; Hou, K. Spatial dispersion-free spectral beam combining of high power pulsed Yb-doped fiber lasers. In Proceedings of the Conference on Lasers and Electro-Optics/Quantum Electronics and Laser Science Conference and Photonic Applications Systems Technologies, San Jose, CA, USA, 4-9 May 2008.

13. Eastman, J.M. Scattering by all-dielectric multilayer bandpass filters and mirrors for lasers. Phys. Thin Films 1978, 10, 167.

14. Born, M.; Wolf, E. Principles of Optics: Electromagnetic Theory of Propagation, Interference and Diffraction of Light; Elsevier: Amsterdam, The Netherlands, 2013.

15. Meinel, K.; M Schindler, K. Scanning tunneling microscopy study on the preparation and characterization of zirconium oxide islands on Ag (100). Surf. Sci. 2002, 515, 226-234. [CrossRef]

16. Vidal, B.; Vincent, P. Metallic multilayers for x rays using classical thin-film theory. Appl. Opt. 1984, 23, 1794-1801. [CrossRef]

17. Prudnikov, I.R. Theoretical modeling of hard X-ray surface modes in a periodic multilayer. Opt. Commun. 2008, 281, 113-120. [CrossRef]

18. Kim, D. The influence of Au thickness on the structural, optical and electrical properties of $\mathrm{ZnO} / \mathrm{Au} / \mathrm{ZnO}$ multilayer films. Opt. Commun. 2012, 285, 1212-1214. [CrossRef]

19. Bennett, H.E.; Porteus, J.O. Relation between surface roughness and specular reflectance at normal incidence. JOSA 1961, 51, 123-129. [CrossRef]

20. Tabatabaeenejad, A.; Moghaddam, M. Bistatic scattering from three-dimensional layered rough surfaces. IEEE Trans. Geosci. Remote Sens. 2006, 44, 2102-2114. [CrossRef]

21. Brown, G.C.; Celli, V. Vector theory of light scattering from a rough surface: Unitary and reciprocal expansions. Surf. Sci. 1984, 136, 381-397. [CrossRef]

22. Carniglia, C.K. Scalar scattering theory for multilayer optical coatings. Opt. Eng. 1979, 18, 104-115. [CrossRef]

23. Hou, H.; Shen, J. Stratified-Interface Scattering Model for Multilayer Optical Coatings. Acta Opt. Sin. 2006, 26, 1102.

24. Chen, F.; Ma, J. $10 \mathrm{~kW}$-level spectral beam combination of two high power broad-linewidth fiber lasers by means of edge filters. Opt. Express 2017, 25, 32783-32791. [CrossRef]

25. Kaiser, T.; Flamm, D. Complete modal decomposition for optical fibers using CGH-based correlation filters. Opt. Express 2009, 17, 9347-9356. [CrossRef]

26. Schmidt, O.A.; Schulze, C. Real-time determination of laser beam quality by modal decomposition. Opt. Express 2011, 19, 6741-6748. [CrossRef]

27. Schulze, C.; Naidoo, D. Wavefront reconstruction by modal decomposition. Opt. Express 2012, 20, $19714-19725$. [CrossRef]

(C) 2019 by the authors. Licensee MDPI, Basel, Switzerland. This article is an open access article distributed under the terms and conditions of the Creative Commons Attribution (CC BY) license (http://creativecommons.org/licenses/by/4.0/). 